\section{$\mathbf{m} / \mathbf{S}$}

médecine/sciences $1995 ; 11$ : 9-12

\title{
THÉRAPIE GÉNIQUE : LE TEMPS D'UN PREMIER BILAN
}

e premier concept associé à la notion de thérapie génique était celui de la réparation des gènes dont l'altération est responsable de maladies. Puis, très rapidement, il est apparu qu'une telle réparation des gènes altérés n'était pas un objectif facile à atteindre alors que l'apport d'un gène fonctionnel dans des cellules dont le gène homologue n'est pas fonctionnel est nettement plus aisé. Dès lors, la thérapie génique devenait l'utilisation du gène comme un nouveau type de médicament [1]. Cette vision devait rapidement déboucher sur des indications débordant largement le cadre des maladies génétiques puisqu'un gène-médicament peut, en principe, remplacer n'importe quel médicament protéique dont il commandera alors la synthèse et la délivrance dans l'organisme même qu'il s'agit de traiter. Alors que les premiers essais de thérapie génique chez l'homme ont maintenant cinq ans, qu'ils sont aujourd'hui au nombre de 150 enrôlant plus de 1000 personnes (voir l'article de T. Valère, $p .73$ de ce numéro [2]), la roue a continué de tourner et il apparaît que, compte tenu des considérables difficultés rencontrées, ce sont des maladies non génétiques, bénéficiant actuellement de médicaments protéiques biologiquement très actifs en administration générale ou locale, qui pourraient être les premières cibles d'une thérapie génique réellement curatrice, alors que les perspectives de traitement par thérapie génique de l'immense majorité des maladies génétiques restent extrêmement lointaines.

\section{Le bon gène à la bonne activité}

La première exigence en thérapie génique est de disposer du bon gène. Dans beaucoup de maladies héréditaires, la question de la nature du bon gène ne se pose pas puisqu'il s'agit de celui dont la mutation est responsable de la maladie. Encore faut-il s'assurer que son niveau de fonctionnement est suffisant pour obtenir un résultat thérapeutique et, à l'inverse, ne risque pas d'entraîner l'apparition d'une nouvelle symptomatologie. Cette dernière éventualité est à prendre en considération lorsque le gène muté code pour une sous-unité d'une protéine hétéropolymérique, puisque la quantité des sous-unités non intéressées par la mutation constitue alors un facteur limitant ; l'hyperexpression du transgène thérapeutique pourrait donc faire apparaître un excès de formes homopolymériques anormales toxique pour la cellule. Ce problème est bien illustré par les thalassémies où un excès de synthèse d'une des chaînes de globine conduirait à la formation d'homodimères et d'homotétramères instables lésant les globules rouges. Certaines maladies 
lysosomales, telles que la maladie de Tay-Sachs, constituent d'autres exemples de cette situation (voir l'article de L. Poenaru, p. 35 de ce numéro [3]). Il peut également se faire qu'il soit indispensable de soumettre le niveau de transcription du gène thérapeutique à un étroit contrôle en fonction de l'état physiologique et de la spécificité tissulaire. Par exemple, un transgène insuline transféré dans des cellules afin de traiter le diabète sucré devrait, naturellement, être soumis à un contrôle en fonction de la glycémie. Dans d'autres cas, le gène d'activité thérapeutique optimale n'a pas encore été trouvé. Ainsi en va-t-il du cancer, où les stratégies actuelles de transfert de gènes codant pour des stimulants de l'immunité, pour des produits toxiques ou pour des anti-oncogènes ont été validés plus sur des modèles de tumeurs greffées chez l'animal immunotolérant, dont on sait qu'ils donnent souvent des résultats flatteurs, que sur des tumeurs spontanées animales ou humaines ([4] et voir l'article de P. Mannoni, p. 68 de ce numéro, [5]). De même, les perspectives d'utilisation de la thérapie génique dans les maladies neurodégénératives dépendra de la possession de gènes codant pour des facteurs neurotrophiques dont l'efficacité potentielle dans les maladies considérées aura véritablement été démontrée [6].

\section{Le bon vecteur}

Tout pharmacologue confronté quotidiennement au difficile problème de la biodisponibilié insuffisante de produits dont l'efficacité in vitro a été démontrée sait bien que les problèmes de galénique sont souvent les plus difficiles à surmonter. Par exemple, les peptides et les oligonucléotides sont à l'évidence de puissants réactifs entre les mains des expérimentateurs [7]; ils ne sont cependant pas encore des médicaments largement utilisés car le problème de leur pénétration intracellulaire sous une forme active n'a pas été bien résolu. On ne peut donc s'étonner de ce que ces difficultés existent également pour des molécules aussi complexes que des gènes. Ce

l'optique d'une utilisation de gènes comme matériel de vaccination. Dans ce cas, en effet, une très faible efficacité de pénétration intracellulaire peut être suffisante et on a probablement le choix quant aux sites d'administration. De ce fait, c'est probablement dans ce domaine de la vaccination génique que les perspectives d'utilisation médicale du transfert de gènes sont le plus crédibles à court et moyen termes. Cependant, personne ne peut s'attendre à ce que toutes les difficultés proprement immunologiques liées à la vaccination soient résolues par un coup de baguette magique du fait de l'utilisation de ce nouveau matériel vaccinal.

Dans l'immense majorité des autres indications de la thérapie génique, le gène médicament devra être «vectorisé » vers sa cible. Le vecteur utilisé peut être viral ou non viral. Trois vecteurs viraux ont été jusqu'à présent particulièrement étudiés : les rétrovirus, les adénovirus et les AAV (adenoassociated virus); d'autres vecteurs viraux en sont encore à un stade plus en amont de leur développement, par exemple les virus herpès. Les vecteurs adénoviraux poseront probablement toujours des problèmes pour des thérapies géniques très prolongées, a fortiori définitives. En effet, le caractère non réplicatif de leur génome et sa position extrachromosomique ne permettent pas de supposer que l'on pourra obtenir des expressions stables pendant plusieurs années. Aujourd'hui, ces vecteurs sont, de plus, lourdement handicapés par leur immunogénicité résiduelle, limitant à quelques semaines l'efficacité des transgènes thérapeutiques qu'ils véhiculent [8]. Cependant, des modifications génétiques complémentaires du génome viral de ces vecteurs, réalisées aujourd'hui par plusieurs équipes à travers le monde, devraient permettre de pallier, au moins en partie, cette difficulté. Quoi qu'il en soit, immunogénicité et instabilité, associées à une trop faible infectiosité dans les conditions pathologiques réelles, expliquent probablement le caractère extrêmement décevant des essais de thérapie génique de la mucoviscidose réalisés en utilisant ce type de vecteur ([9], voir l'article de A. Pavirani et al., p. 25 de ce numéro [10]). En revanche, seule une très bonne infectiosité pour les cellules tumorales est requise pour que l'adénovirus constitue la base d'un bon vecteur de thérapie génique du cancer [5]. La difficulté principale est donc ici de mettre, dans ce bon vecteur, ... un bon gène.

Les vecteurs AAV, après quelques promesses, s'avèrent aujourd'hui d'utilisation bien difficile. En effet, et malgré quelques publications optimistes, ce vecteur reste très difficile à obtenir à un titre élevé. De plus, et surtout, il est apparu qu'il n'était efficace qu'associé à des virus auxiliaires réplicatifs, adénovirus ou virus herpès.

Les vecteurs rétroviraux restent l'outil de base pour obtenir le transfert et une expression stable d'un transgène thérapeutique. Cependant, les progrès faits pour pallier leurs principaux inconvénients restent lents. Les limitations à leur utilisation large restent aujourd'hui : les faibles titres viraux obtenus, leur inefficacité sur des cellules qui ne se divisent pas et l'extrême réticence avec laquelle on envisage de les utiliser directement in vivo, pour des raisons de sécurité liées à l'insertion au hasard de l'ADNc proviral dans l'ADN chromosomique.

Quant aux vecteurs non viraux, constitués avant tout de lipides cationiques (voir l'article de J.-P. Behr, p. 56 de ce numéro [11]), ils restent aujourd'hui d'efficacité variable selon les cellules, et probablement encore faible en administration directe dans l'organisme. L'utilisation de ces vecteurs par voie intrabronchique, également dans le cadre d'un essai de thérapie génique de la mucoviscidose, confirme que beaucoup de progrès restent à réaliser pour conférer à ce type de formulation une efficacité in vivo acceptable [12].

\section{La bonne voie d'administration}

Si nous supposons résolues ces questions d'une construction génique optimale et d'un vecteur sûr et efficace, reste à régler le très difficile problème de la voie d'administration. Dans le cancer, les administrations locales risquent d'être souvent insuffisantes pour une maladie dont le pouvoir métastasant représente une 
grande partie de la gravité. Dans la mucoviscidose, des incertitudes persistent quant à la vraie nature des cellules bronchiques et alvéolaires qu'il faudrait corriger et quant à la voie d'administration qui permettrait le mieux de les atteindre [13]. Dans les myopathies, l'administration intramusculaire directe paraît peu crédible pour des affections intéressant tous les muscles. De même, nous n'avons aujourd'hui aucune idée claire sur la manière dont il serait possible de transférer un gène dans une proportion notable de motoneurones ou d'autres cellules disséminées dans le système nerveux central. La question de la voie d'administration est d'ailleurs de même nature que celle de la vectorisation puisqu'il s'agit dans les deux cas, en définitive, d'aboutir à une réelle biodisponibilité du gène introduit, ce qui confirme que les problèmes aujourd'hui prédominants de la thérapie génique sont bien de nature galénique.

\section{La bonne indication}

Dans le cas des maladies génétiques incurables, seule se pose la question de la faisabilité, et non pas de l'indication. Cependant, ce problème de l'indication est d'actualité pour toutes les formes de thérapie génique qui consistent à remplacer une protéine thérapeutique par le gène en commandant la synthèse in vivo, ou lorsque le choix peut être fait entre une greffe d'organe ou de cellules et une thérapie génique. Par exemple, si les progrès réalisés permettaient bientôt de proposer une délivrance in situ d'insuline par thérapie génique chez des diabétiques $\left(\mathrm{m} / \mathrm{s} n^{\circ} 7\right.$, vol. $11, p$. 1049), d'érythropoiétine chez des thalassémiques ou des malades souffrant d'anémie $\left(\mathrm{m} / \mathrm{s} n^{\circ} 3\right.$, vol. 11, p. 495), d'enzymes diverses chez des malades souffrant de déficit enzymatique, cette stratégie devrait être soigneusement évaluée en comparaison avec les autres possibilités thérapeutiques : injection de la protéine recombinante correspondante, implantation de cellules allogéniques ou xénogéniques microencapsulées, micropompes, etc. Dans cette évaluation interviendront les notions d'acceptabilité pour les malades, de sécurité, d'efficacité, de tolérance à moyen et long termes... et de coût.

\section{Un contexte brouillé}

Ainsi apparaît-il que des efforts substantiels restent à accomplir avant de transformer la thérapie génique en une thérapie crédible pour le futur, et que cette crédibilité reste variable selon les maladies, probablement plus grande pour des maladies curables par un traitement bref que pour des maladies génétiques. Cela n'a pas empêché une activité frénétique de se développer autour de la thérapie génique, aussi bien médicale et industrielle que médiatique. Pour les industriels, l'analyse prédominante est que, dans l'avenir, seules des thérapeutiques vraiment innovantes pourront s'imposer, compte tenu des efforts consentis par les différents États pour diminuer les dépenses de santé. Malgré les incertitudes, il paraît évident que certaines affections, aujourd'hui non contrôlées par la thérapeutique classique, seront améliorées par des thérapies géniques. L'investissement dans ce domaine de nombreux grands groupes pharmaceutiques et biotechnologiques est donc généralement lucide et raisonné. En revanche, l'activité expérimentale clinique est un peu désordonnée et obéit à des schémas stratégiques qui ne doivent parfois rien aux impératifs de la recherche thérapeutique. De très nombreuses petites compagnies de biotechnologie, que l'on désigne souvent sous le terme start-ups, ont choisi le domaine de la thérapie génique pour «se faire les dents". Pour ces sociétés, l'accès à l'essai clinique équivaut pratiquement à la conquête du Saint-Graal : ces essais, promis aux investisseurs grâce aux fonds desquels ces sociétés sont créées et fonctionnent, justifient l'appel à des investissements supplémentaires, en partie indépendamment de leurs résultats. Ainsi, certains essais cliniques sans réelles perspectives scientifiques ou thérapeutiques ont-ils été réalisés à la seule fin de sauvegarder la valeur en bourse des actions de certaines firmes. D'autres fois, la pression médiatique autour de ces essais thérapeutiques a probablement expliqué que des équipes clinicobiologiques passent à l'essai clinique un peu avant que cela ne soit justifié par les essais précliniques. C'est également cette atmosphère entourant le développement de la recherche expérimentale sur la thérapie génique qui explique le déchet considérable, en terme de qualité, des publications consacrées à ce champ. En réalité, le nombre de publications d'un faible niveau scientifique et peu convaincantes publiées en ce domaine, même dans de bons journaux, est étonnant. Ces critiques ne peuvent cependant pas être faites au très petit nombre d'articles présentant enfin les résultats d'essais cliniques dans la mucoviscidose [9], le déficit immunitaire associé à la carence en adénosine désaminase [14, 15], l'hypercholestérolémie familiale [16] et les gliomes inopérables. La bonne qualité de ces publications permet d'observer que, s'il existe quelques données encourageantes, les résultats globaux de ces essais sont cependant très éloignés du succès et, à l'exception peut-être du traitement des malades atteints de déficit en adénosine désaminase, auraient même pu être appelés des échecs si l'essai thérapeutique avait concerné tout autre type de médicament. Dès lors, on ne peut manquer de soulever la légitimité, pour le futur, de certains essais extrêmement invasifs (par exemple, hépatectomie partielle puis injection intraportale de plusieurs milliards de cellules chez des malades atteints d'hypercholestérolémie familiale) tant que les protocoles proposés resteront aussi probablement peu efficaces. Cette analyse critique et ces questions sont d'ailleurs exactement du même ordre que celles du rapport que vient de remettre un comité d'experts, conduit par Arno Motulsky et Stuart Orkin, à Harold Varmus, directeur du NIH [17] : la thérapie génique est un concept tout nouveau ; elle est prometteuse pour le traitement de certaines maladies dans le futur, mais les difficultés à résoudre restent considérables, ce qui est en soi une motivation amplement suffisante pour poursuivre avec ardeur et exigence les travaux de développement de cette méthode thérapeutique du futur. Cela exige de concentrer la majorité des efforts sur les questions de galénique génique, c'est-à-dire de vectorisation et d'étude des voies d'administration, allant à l'essai clinique chaque fois que cela 
sera nécessaire, mais rien que quand cela sera nécessaire, dans le respect de l'éthique scientifique et des malades. Dans ce contexte, médecine/sciences a désiré consacrer pratiquement un numéro spécial à la thérapie génique, afin de montrer ce que pourrait être dans l'avenir de la bonne science mise au service d'un bel objectif

\section{RÉFÉRENCES}

1. Kahn A. Thérapie génique, l'ADN médicament. Paris : John Libbey Eurotext, 1993.

2. Valère $T$. Thérapie génique : le point sur les essais cliniques. médecine/sciences 1996 ; $12: 73-83$

3. Poenaru L. Thérapie génique des maladies lysosomales. médecine/sciences $1996 ; 12$ : 35- 46 .

4. Fernandez N, Abina M, Duffour M, Haddada $\mathrm{H}$. Vaccination antitumorale et thérapie génique. médecine/sciences 1995 ; 11 : 97583

5. Mannoni P. La thérapie génique du cancer : désir mythique ou réalité therapeutique de demain ? médecine/sciences 1996 ; 12 : 6872 .

6. Henderson C. L'avenir thérapeutique des facteurs neurotrophiques dans les maladies neurodégénératives. médecine/sciences 1995 ; $11: 1067-9$

7. Hélène C, Saison-Behmoaras ? La stratégie antisens : nouvelles approches thérapeutiques. médecine/sciences $1994 ; 10$ : 253-73.

8. Gilgenkrantz H. L'infection intrathymique néonatale d'adénovirus recombinant: une voie de tolérisation. médecine/sciences $1995 ; 11$ : 1371-2.

9. Crystal R, McElvaney M, Rosenfeld M, Chu C, Mastrangeli A, Hay J, Brody S, Jaffe H, Eissa N, Danel C. Administration of an adenovirus containing the human CFTR cDNA to the respiratory tract of individuals with cystic fibrosis. Nature Genet 1994 ; 8 : 42-50.

10. Pavirani A, Schatz C, Mehtali M. Thérapie génique de la mucoviscidose par transfert adénoviral du gène CFTR. médecine/sciences $1996 ; 12$ : 25-33.
11. Behr JP. L' éponge à protons : un moyen d'entrer dans une cellule auquel les virus n'ont pas pensé. médecine/sciences 1996 ; 12 : 56-9.

12. Caplen NJ, Alton EWFW, Middelton PG, Dorin JR, Stevenson BI, Gao X, Durham SR, Jeffery PK, Hodson ME, Coutelle C, Huang L, Porteous DJ, Williamson R, Geddes DM (St Mary's Hospital, London). Liposomemediated CFTR gene transfer to the nasal epithelium of patients with cystic fibrosis. Nature Med 1995 ; 1 : 39-46.

13. Merten M, Becq F. Mucoviscidose : vers quelles cellules pulmonaires faut-il orienter la recherche fondamentale et clinique? $m e ́-$ decine/sciences $1995 ; 11: 1442-6$.

14. Bordignon C, Notarengelo LD, Nobili N, Ferrari G, Casorati P, Panina P, Mazzolari E, Magggoni D, Rossi C, Servida P, Ugazio AG, Mavilio F. Gene therapy in peripheral blood lymphocytes and bone marrow for ADA-immunodeficient patients. Science 1995 ; 270 : 470-5.

15. Blaese RM, Culver KW, Miller AD, Carter CS, Fleisher T, Clerici M, Shearer G, Chang L, Chiang Y, Tolstoshev P, Greenblatt JJ, Rosenberg SA, Klein H, Berger M, Mullen CA, Ramsey WJ, Muul L, Morgan RA, Anderson WF. T-lymphocyte-directed gene therapy for ADA-SCID : initial trial results after 4 years. Science $1995 ; 270$ : 475-80.

16. Grossman M, Rader DJ, Muller DWM, Kolansky DM, Kozarsky K, Clark III DJ, Stein EA, Lupien PJ, Brewer Jr HB, Raper SE, Wilson J. A pilot study of ex vivo gene therapy for homozygous familial hypercholesterolemia. Nature Med $1995 ; 1$ : 1148-54.

17. Marshall E. Less hype, more biology needed for gene therapy. Science 1995 ; 270 : 1751 .

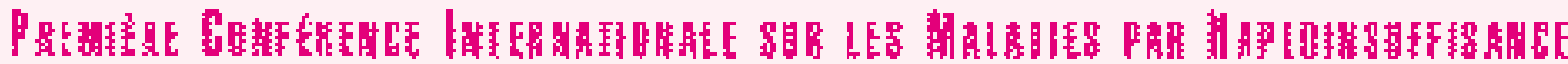

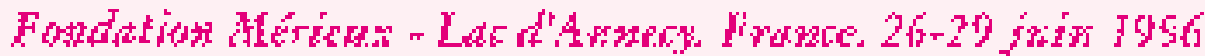

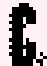

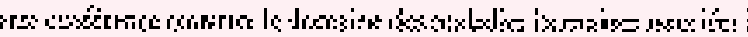

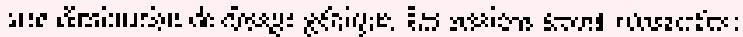

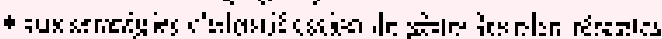

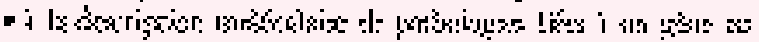

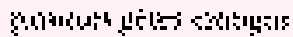

- 3

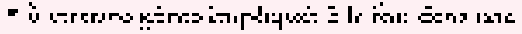

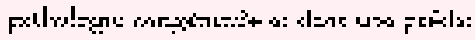

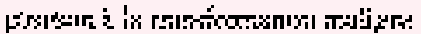

\section{fromita sientigisop}

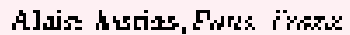

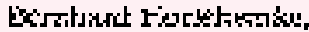

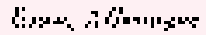

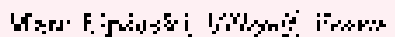

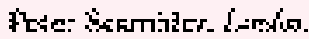

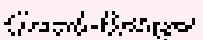

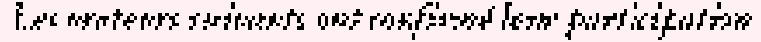

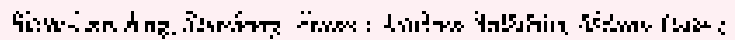

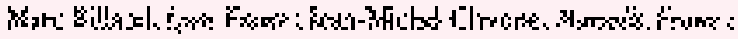

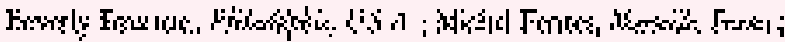

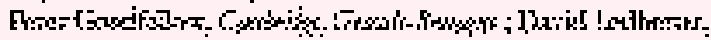

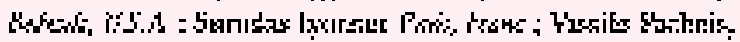

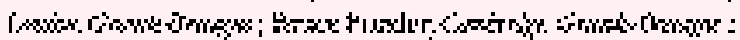

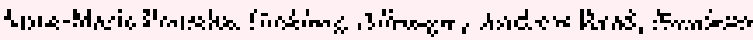

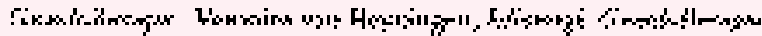

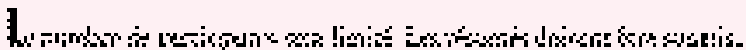

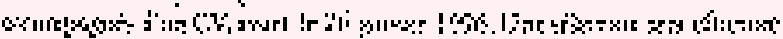

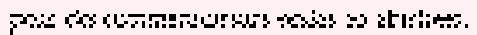

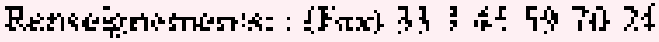

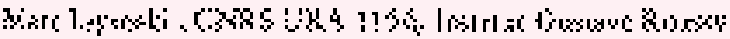

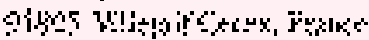

